\title{
From the Heterotic String Quartet to the Cosmic Dark Matter, Dark Energy and Ordinary Energy Symphony
}

\author{
Mohamed S. El Naschie \\ Dept. of Physics, Faculty of Science, University of Alexandria, Alexandria, Egypt \\ Email address: \\ Chaossf@aol.com \\ To cite this article: \\ Mohamed S. El Naschie. From the Heterotic String Quartet to the Cosmic Dark Matter, Dark Energy and Ordinary Energy Symphony. \\ American Journal of Astronomy and Astrophysics. Vol. 5, No. 2, 2017, pp. 21-24. doi: 10.11648/j.ajaa.20170502.12
}

Received: March 6, 2017; Accepted: March 24, 2017; Published: April 10, 2017

\begin{abstract}
The main novel part of the paper is to identify the 16 extra bosonic dimensions of the Heterotic string theory with the negative signature of K3 Kähler manifold and these in turn are the source of pure dark energy. Guided by the basic ideas and structure of heterotic string theory we establish an energy density triality, which add together to the theoretically expected energy density based on Einstein's relativity. Thus Einstein's famous formula $\mathrm{E}=\mathrm{mc}^{2}$ is found in integer approximation to be the sum of three sectors, namely $E=\left(\frac{1+5+16}{22}\right) m c^{2}$ where $E(O) \cong m c^{2} / 22$ is the ordinary energy density, $E(D M) \cong(5 / 22) m c^{2}$ is the dark matter energy density and $E(P D) \cong(16 / 22) m c^{2}$ is the pure dark energy density where $\mathrm{m}$ is the mass, $\mathrm{c}$ is the velocity of light and 16 is the number of heterotic strings extra bosons. We demonstrate further that strictly speaking dark matter is weakly coupled with pure dark energy and that while dark matter and ordinary energy are attracting in the conventional way, pure dark energy has an opposite sign similar to the extra 16 dimensions of heterotic strings making it act effectively in the opposite direction in conformity with the negative sign and magnitude of the corresponding K3 Kähler manifold.
\end{abstract}

Keywords: Heterotic Strings, David Gross 16 Extra Dimensions, Pure Dark Energy, Dark Matter, Kaluza-Klein Theories, Fractal Spacetime, E-infinity Theory

\section{Introduction}

The main point of the present multifaceted paper is to link the signature of the $\mathrm{K} 3$ Kähler manifold to the 16 extra bosonic dimensions of Heterotic strings and show the result explains the contra intuitive effect of pure dark energy. To avoid the mind boggling questions surrounding the true meaning of dimensionality in general [1-14] and how it is used in the present work in particular, we stress the following: We are assuming that we are at the very beginning of say "creation" and at such a high energy scale corresponding to extremely small distances where in this spacetime "primordial soup" so to speak, the distinction between symmetry, dimensions and quantum particles almost vanishes [15-17]. Thus whenever we say particles here, we mean pre-quantum particle-like states which will result from symmetry breaking of a corresponding Lie group of certain dimensionality [15-19]. Something similar will be assumed with regard to the word wave with which we mean prequantum wave of the pre-quantum particle [19-28].

Having clarified our "somewhat fuzzy" terminology we turn our attention to the main theory upon which the present model and analysis is based, namely the heterotic string theory $[14,17,18]$. The essence of this theory is lucidly explained in many papers and text books notably by the creators of the theory, namely a group around Nobel Laureate David Gross $[17,18]$ which is often fondly referred to as the "Princeton quartet" $[17,18]$. Never the less because we will present this theory in the next section with a minor twist here and there, we felt it pertinent to give here a brief account of the quintessential idea behind heterotic string theory seen from our "pure dark energy and dark matter" explanation view point [20-27]. 


\section{Heterotic String Theory for Dark Energy Via the Signature of K3- Kähler}

We think it is fair to say that what David Gross [18] had in mind when he and his group started work on a new heterotic string theory was simply to combine the Green-Schwarz so called Type II, at the time a new ten dimensional superstring theory with the relatively older 26 dimensional bosonic string theory [13-18]. This line of thinking, in the best tradition of unification simplicity, started from the seemingly harmless and deceptively trivial equation [13-18]

$$
26-16=10
$$

However in this way Gross and his three musketeers Jeffrey Harvey, Emil Martinec and Ryan Rohm, all from Princeton [13-18], combined a ten dimensional fermionic field moving to the right with a twenty six dimensional field moving to the left $[17,18]$. Again an embarrassingly trivial rearrangement of the preceding equation clearly shows that a consistent description of the relevant bosonic filed requires 16 extra coordinates [13-18]. It is those 16 extra coordinates corresponding to 16 dimensions or bosonic pre-quantum particles which will play a crucial role in the next section regarding pure dark energy as distinct from dark matter and ordinary energy [20-24]. We note on passing, without going into detail, that the vital 16 extra bosonic spacetime dimensions need compactification and result in a gauge group $U(1)^{16}$ which when broken gives us the carrier of pure dark energy [11]. In turn this behavior is linked to the signature of the K3 Kähler manifold of the theory.

\section{Pure Dark Energy, Dark Matter and Ordinary Energy from Heterotic String Theory}

Let us start from the old 26 dimensional bosonic string theory once more $[10,13,17,18]$. For sure the 26 must include our $3+1=4$ classical spacetime or Einstein's 4D spacetime. In both cases it is reasonable to assume that we cannot see the remaining 26-4=22 dimensions and can regard them as compactified in one way or another similar to how we got used to think of the Kaluza-Klein 5D theory [22]. The next step in our simple scheme is to think of these 22 quasi dimensions as a sort of pre-quantum messenger particles [20-24]. Having accepted this move let us subdivide these 22 messenger particles to 6 plus 16 bosons [20-27]. The reader has no doubt guessed our disarmingly simple intention to link heterotic strings with the three sectors that make up the entire energy of the cosmos. We are all familiar with the classical photon $\gamma$ similar to Einstein when he devised his special theory of relativity and found his beauty $\mathrm{E}=\mathrm{mc}^{2}$ [23] where $\mathrm{E}$ is the energy, $\mathrm{m}$ is the mass and $\mathrm{c}$ is the speed of light of the good old photon $\gamma$ [20-27]. Furthermore in the meantime we know much more than Einstein, namely that there are 3 more photon- like messenger particles which possess mass unlike the massless classical photon of special relativity and everyday life [13]. In addition we also know of two more bosons related to gravity and mass. Since our analysis needs no more than dimensions or at most pre-quantum particles, you could think of these two bosons as one Higgs and one graviton $[14,17,18]$. That way we need only to believe in the 16 extra bosons of heterotic strings to find that we have our hidden $1+5+16=22$ dimensions or the corresponding quasi pre-quantum bosonic particles. Not only that but we now have the wonderful opportunity of relating these 16 Princeton quartet bosons, moving in a different direction to the rest, to the pure dark energy section [20-27]. Needless to say, we needed the opposite sign to be a negative one disparately in order to justify the fact that pure dark energy can make sense in connection with the accelerated expansion of the universe only if it were a repulsive rather than attractive energetic force [20-25]. This is as near as we can come to anti gravity [20-24].

From the above we can see that $\mathrm{E}=\mathrm{mc}^{2}$ which represents the maximal energy density in the universe can be subdivided into three parts in a way delightfully consistent with the theoretical and experimental observational work on dark energy. To make our point even clearer let us write Einstein's maximal energy density as [24, 27]

$$
\begin{aligned}
E(\max ) & =\gamma(\max ) m c^{2} \\
& =\left(\frac{22}{22}\right) m c^{2} \\
& =m c^{2}
\end{aligned}
$$

In other words the maximal density "Lorentzian" factor is $[24,27]$

$$
\begin{aligned}
\gamma(\max ) & =22 / 22 \\
& =1
\end{aligned}
$$

Within our simple scheme the rest is now crystal clear and may be reasoned in the following elementary fashion.

The classical measurable ordinary energy is obviously connected to the ordinary energy of the classical photon, namely [24, 27]

$$
\begin{aligned}
\gamma(O) & =(\gamma=1) / 22 \\
& =0.04545 \\
& \simeq 4.5 \%
\end{aligned}
$$

of the total energy in full agreement with the numerous modern cosmic measurements and observations [20-24]. Second the not directly observed dark matter density, the effect of which is only inferred from observation connected to non-classical gravitational measurement may now reasonably be attributed to the five pre-quantum messenger particles discussed earlier on. That way we can set the dark matter energy density to be $[24,27]$ 


$$
\begin{aligned}
\gamma(D M) & =\frac{5}{22} \\
& =0.227 \\
& \simeq 22.7 \%
\end{aligned}
$$

of the total energy of the universe. Again this is in excellent agreement with cosmic measurements [20-27].

Finally, and most importantly, we can now determine the pure dark energy density as being the result of the 16 extra bosons of the heterotic string energy which we reinterpret here as part of the contra intuitive topology of a K3 Kähler with a signature equal to 16 but with a negative sign. That way we find not only [24, 27]

$$
\begin{aligned}
\gamma(P D) & =\frac{16}{22} \\
& =0.727 \\
& \simeq 72.7 \%
\end{aligned}
$$

of the total energy but also knowing that it has an opposite sign resolves the question of repulsive rather than attractive effect of pure dark energy as distinct from pure dark matter $[12,22,24]$.

Unfortunately the preceding simple calculation, although very accurate when compared to measurements and observation, does not show how dark matter and pure dark energy are coupled. To do that we have to give an exact solution and such an exact solution requires the exact Einfinity theory [19] of dark energy [20-27]. In this theory the dark energy is the energy of the quantum wave represented by the empty set and ordinary energy is the energy of the quantum particle represented by the zero set $[20,23]$. To subdivide the quantum wave into pure dark energy and dark matter and see how they are coupled, we must use the exact transfinite dimension of the bosonic string theory [22]. This is $26+\mathrm{k}$ rather than simply 26 [24]. This $\mathrm{k}$ is a small value equal to $\phi^{3}\left(1-\phi^{3}\right)=0.1803398$ where $\phi=(\sqrt{5}-1) / 2$ and was conjectured to relate to the 'tHooft (not yet discovered) renormalon particle [21].

To sum up the preceding section, we saw that $\mathrm{E}=\mathrm{mc}^{2}$ of Einstein may be seen as a theoretical maximal energy density for the cosmos because a system with one degree of freedom is maximally stiff and its Eigen value must be the largest possible [28]. Linking this maximal density to the basic structure of heterotic string theory we were lead to the following trisectional division, namely $[21,24]$

$$
\begin{aligned}
E & =\frac{\gamma^{o}+\left(W^{+}+W^{-}+Z^{o}+H+G\right)+16 \text { extra bosons }}{D^{(26)}-D^{(4)}} m c^{2} \\
& =\frac{1+5+16}{26-4} m c^{2} \\
& =\frac{22}{22} m c^{2} \\
& =E(\text { Einstein })
\end{aligned}
$$

Before any misunderstanding arises regarding the unusual nature of the involved 16 "things", we hasten to say that on the one hand they account for gluon as well as the electroweak gauge fields but on the other hand, within the string itself we have 16 dimensional bosonic fields as stressed by D. Gross. It is the latter aspect that is the carrier of pure dark energy. The exact transfinite version of the above was discussed using a different mathematical framework elsewhere and it is sufficient here to give the final result and refer the reader to the literature $[20,22,24]$. In short we just need to replace 26 by $26+\mathrm{k}$ and 16 by $16+\mathrm{k}$. That way we find that 22 becomes $(26+k)-4=22+k$ so that our basic triadic decomposition of $\mathrm{E}=\mathrm{mc}^{2}$ becomes [24]

$$
E=\left(\frac{1}{22+k}+\frac{5-\Delta}{22+k}+\frac{16+k+\Delta}{22+k}\right) m c^{2}
$$

where $\Delta=\left(8+k^{2}\right) / 100$ is the coupling between pure dark energy and dark matter. Evaluating the above the result is a firm confirmation of Einstein's maximal energy density [24]

$$
\begin{aligned}
E & =\left(\frac{22+k}{22+k}\right) m c^{2} \\
& =m c^{2}
\end{aligned}
$$

which means the coupling term $\Delta=\left(8+k^{2}\right) / 100$ cancels out and earlier results obtained using different methods are confirmed [24].

\section{Conclusion}

The topology of a K3 Kähler manifold is characterized by a negative signature equal minus 16 among other invariants which we link to the space time formed by the Hetrotic strings with its famous 16 bosonic extra strings. These 16 extra bosons of heterotic strings seems to be an excellent explanation of pure dark energy as distinct from dark matter or the dark energy of the quantum wave which lumps pure dark energy and dark matter together to make about $95.5 \%$ of the total energy density of the universe. Adding the ordinary energy to this one finds the total energy density which is equal to that of Einstein. However in the present work we can discriminate between dark energy and pure dark energy in an accurate and plausible way provided we first believe in the cosmic measurements and observation and second in the consistency and correctness of the heterotic string theory. The present author firmly believes in the cosmic measurements and observations and particularly in accelerated cosmic expansion. On the other hand we have the fact that heterotic strings 16 extra bosonic quasi particles not only account for the right measurements but also give the right sign for the repulsive force of pure dark energy which can explain the accelerated cosmic expansion. Due to these facts the author feels that heterotic strings, unconventional as it may seem, must be a substantially accurate description of not only high energy physics but also quantum cosmology and astrophysics. At a minimum heterotic string theory is one 
of the truly good models to account for pure dark energy and accelerated cosmic expansion.

\section{Acknowledgement}

Without the research of the 'Princeton string quartet' lead by Prof. D. Gross, this paper could never be possible. Although this is self evident it had to be said in gratitude.

\section{References}

[1] R. Engelking: Theory of Dimensions Finite and Infinite. Heldermann Verlag, Lemgo, Germany. 1995.

[2] N. J. Wilenkin: Unterhaltsame Mengenlehre Verlag Havri Deutsch. Leipzig, Germany. 1973.

[3] M. S. El Naschie: Infinite dimensional Branes and the Einfinity toplogy of heterotic superstrings. Chaos, Solitons \& Fractals, 2001, 12, pp. 1047-1055.

[4] L. Marek-Crnjac: Partially ordered sets, transfinite topology and the dimension of Cantorian-fractal spacetime. Chaos, Solitons \& Fractals, 42 (3), 2009, pp. 1796-1799.

[5] L. Marek-Crnjac, G. Iovane, S. I. Nada and Ting Zhang: The mathematical theory of finite and infinite dimensional topological spaces and its relevance to quantum gravity. Chaos, Solitons \& Fractals, 42 (4), 2009, 1974-1979.

[6] S. I. Nada: Density manifolds, geometric measures and high energy physics in transfinite dimensions. Chaos, Solitons \& Fractals, 42 (3), 2009, pp. 1339-1541.

[7] S. I. Nada: On the mathematical theory of transfinite dimensions and its application in physics. Chaos, Solitons \& Fractals, 42 (1), 2009, pp. 530-531.

[8] Guo-Cheng $\mathrm{Wu}$ and Ji-Huan He: On the Menger Urysohn theory of Cantorian manifolds and transfinite dimensions in physics. Chaos, Solitons \& Fractals, 42 (2), 2009, pp. 781783.

[9] Sudhanva Joshi: Theory of quantum relativity. Journal of Quantum Information Science, 6 (4), 2016, pp. 249-262.

[10] M. Kaku: Introduction to Superstrings and M-theory. Springer, New York (1999).

[11] T. Ortin: Gravity and Strings. Cambridge University Press, Cambridge, UK. 2004.

[12] L. Marek-Crnjac: Generalized quantum entanglement family in connection to black holes and nanotechnology. Chapter in "Quantum Gravity", Edited by B. Mitchell. Nova Publishers, New York, USA. 2017.

[13] F. D. Peat: Superstrings. Abacus, London, UK. 1992.
[14] J. Polchinski: String Theory Vol. I and Vol. II: Cambridge University Press, Cambridge, 1999.

[15] M. S. El Naschie: From symmetry to particles. Chaos, Solitons \& Fractals, 32 (2), 2007, pp. 427-430.

[16] M. S. El Naschie: Topics in the mathematical physics of Einfinity theory. Chaos, Solitons \& Fractals, 30 (3), 2006, pp. 656-663.

[17] David Gross, J. A. Harvey, E. Martinec and R. Rohm: Heterotic string theory (1). The free Heterotic string. Nuclear Physics B, 256, 1985, pp. 253-284.

[18] David Gross, J. A. Harvey, E. Martinec and R. Rohm: Physical Review Letters, 54 (6), 1985, pp. 502.

[19] M. S. El Naschie: A review of E-infinity theory and the mass spectrum of high energy particle physics. Chaos, Solitons \& Fractals, 19 (1), 2004, pp. 209-236.

[20] Mohamed S. El Naschie: A resolution of cosmic dark energy via quantum entanglement relativity theory. Journal of Quantum Information Science, 2013, 3, pp. 23-26.

[21] Mohamed S. El Naschie: On a new elementary particle from the disintegration of the symplectic 't Hooft-Veltman-Wilson fractal spacetime. World Journal of Nuclear Science and Technology, Vol, 4 (4), 2014, pp. 216-221.

[22] Mohamed S. El Naschie: On a fractal version of Witten's Mtheory. Journal of Astronomy \& Astrophysics, 6 (2), 2016, pp. $135-144$.

[23] A. J. Babchin and M. S. El Naschie: On the real Einstein beauty $\mathrm{E}=\mathrm{kmc}^{2}$. World Journal of Condensed Matter Physics, $6(1), 2016$.

[24] M. S. El Naschie: Kerr black hole geometry leading to dark matter and dark energy via E-infinity theory and the possibility of nano spacetime singularity reactor. Natural Science, 7 (4), 2015, pp. 210-225.

[25] Mohamed S. El Naschie: The looped light of the triple-slit real experiment as a confirmation for the extra dimensions of quantum spacetime and the reality of dark energy. Optical and Photonic Journal, 7 (2), 2017, pp. 19-26.

[26] Mohamed S. El Naschie: Looped light on dark energy. Journal of Quantum Information Science, 7 (1), 2017, pp. 1-5.

[27] Mohamed S. El Naschie: Quantum disentanglement as the physics behind dark energy. Open Journal if Mircophysics, 7 (1), 2017, pp. 1-27.

[28] M. S. El Naschie: Computing dark energy and ordinary energy of the cosmos as a double Eigenvalue problem. Journal of Modern Physics, 6 (4), 2015, pp. 348-395. 